\title{
Regularity of solutions in semilinear elliptic theory
}

\author{
Emanuel Indrei ${ }^{1}$ - Andreas Minne ${ }^{2}$. \\ Levon Nurbekyan ${ }^{3}$
}

Received: 3 March 2016 / Revised: 27 May 2016 / Accepted: 28 June 2016 /

Published online: 8 July 2016

(C) The Author(s) 2016. This article is published with open access at Springerlink.com

Abstract We study the semilinear Poisson equation

$$
\Delta u=f(x, u) \text { in } B_{1} .
$$

Our main results provide conditions on $f$ which ensure that weak solutions of (1) belong to $C^{1,1}\left(B_{1 / 2}\right)$. In some configurations, the conditions are sharp.

Keywords Semilinear elliptic theory · Partial differential equations · Regularity theory

\section{Introduction}

The semilinear Poisson equation (1) encodes stationary states of the nonlinear heat, wave, and Schrödinger equation. In the case when $f$ is the Heaviside function in the $u$ -

\section{Communicated by Neil Trudinger.}

Emanuel Indrei

egi@cmu.edu

Andreas Minne

minne@kth.se

Levon Nurbekyan

levon.nurbekyan@kaust.edu.sa

1 Center for Nonlinear Analysis, Carnegie Mellon University, Pittsburgh, PA 15213, USA

2 Department of Mathematics, KTH Royal Institute of Technology, 10044 Stockholm, Sweden

3 CEMSE Division, King Abdullah University of Science and Technology (KAUST), Thuwal 23955-6900, Saudi Arabia 
variable, (1) reduces to the classical obstacle problem. For an introduction to classical semilinear theory, see $[4,5]$.

It is well-known that weak solutions of (1) belong to the usual Sobolev space $W^{2, p}\left(B_{1 / 2}\right)$ for any $1 \leq p<\infty$ provided $f \in L^{\infty}$. Recent research activity has thus focused on identifying conditions on $f$ which ensure $W^{2, \infty}\left(B_{1 / 2}\right)$ regularity of $u$.

\subsection{The classical theory}

There are simple examples which illustrate that continuity of $f=f(x)$ does not necessarily imply that $u$ has bounded second derivatives: for $p \in(0,1)$ and $x \in \mathbb{R}^{2}$ such that $|x|<1$, the function

$$
u(x)=x_{1} x_{2}(-\log |x|)^{p}
$$

has a continuous Laplacian but is not in $C^{1,1}$ [15]. However, if $f$ is Hölder continuous, then it is well-known that $u \in C^{2, \alpha}$; if $f$ is Dini continuous, then $u \in C^{2}[7,11]$. The sharp condition which guarantees bounded second derivatives of $u$ is the $C^{1,1}$ regularity of $f * N$ where $N$ is the Newtonian potential and $*$ denotes convolution; this requirement is strictly weaker than Dini continuity of $f$.

In the general case, the state-of-the-art is a theorem of Shahgholian [14] which states that $u \in C^{1,1}$ whenever $f=f(x, u)$ is Lipschitz in $x$, uniformly in $u$, and $\partial_{u} f \geq$ $-C$ weakly for some $C \in \mathbb{R}$. In some configurations this illustrates regularity for continuous functions $f=f(u)$ which are strictly below the classical Dini-threshold in the $u$-variable, e.g. the odd reflection of

$$
f(u)=-\frac{1}{\log (u)}
$$

about the origin. Shahgholian's theorem is proved via the celebrated Alt-CaffarelliFriedman (ACF) monotonicity formula and it seems difficult to weaken the assumptions by this method. On the other hand, Koch and Nadirashvili [10] recently constructed an example which illustrates that the continuity of $f$ is not sufficient to deduce that weak solutions of $\Delta u=f(u)$ are in $C^{1,1}$. With all this in mind, we make the following assumption.

Assumption A Let $f=f(x, u)$ be Dini continuous in $u$, uniformly in $x$, and assume it has a $C^{1,1}$ Newtonian potential in $x$, uniformly in $u$.

One of our main results is the following statement.

Theorem 1.1 Suppose $f$ satisfies Assumption A. Then any solution of (1) is $C^{1,1}$ in $B_{1 / 2}$.

Our assumption includes functions which fail to satisfy both conditions in Shahgholian's theorem, e.g.

$$
f\left(x_{1}, x_{2}, t\right)=\frac{x_{1}}{\log \left(\left|x_{2}\right|\right)(-\log |t|)^{p}}
$$


for $p>1, x=\left(x_{1}, x_{2}\right) \in B_{1}$ and $t \in(-1,1)$. The Newtonian potential assumption in the $x$-variable is essentially sharp whereas the condition in the $t$-variable is in general not comparable with Shahgholian's assumption.

The proof of Theorem 1.1 does not invoke monotonicity formulas and is selfcontained. We consider the $L^{2}$ projection of $D^{2} u$ on the space of Hessians generated by second order homogeneous harmonic polynomials on balls with radius $r>0$ and show that the projections stay uniformly bounded as $r \rightarrow 0^{+}$. Although this approach has proven effective in dealing with a variety of free boundary problems $[2,6,8,9]$, Theorem 1.1 illustrates that it is also useful in extending and refining the classical elliptic theory.

\subsection{Singular case: the free boundary theory}

In $\S 4$ we study the PDE (1) for functions $f=f(x, u)$ which are discontinuous in the $u$-variable at the origin.

If the discontinuity of $f$ is a jump discontinuity, (1) has the structure

$$
f(x, u)=g_{1}(x, u) \chi_{\{u>0\}}+g_{2}(x, u) \chi_{\{u<0\}},
$$

where $g_{1}, g_{2}$ are continuous functions such that

$$
g_{1}(x, 0) \neq g_{2}(x, 0), \quad \forall x \in B_{1},
$$

and $\chi_{\Omega}$ defines the indicator function of the set $\Omega$.

Our aim is to find the most general class of coefficients $g_{i}$ which generate interior $C^{1,1}$ regularity.

The classical obstacle problem is obtained by letting $g_{1}=1, g_{2}=0$, and it is wellknown that solutions have second derivatives in $L^{\infty}$ [13]. Nevertheless, by selecting $g_{1}=-1, g_{2}=0$, one obtains the so-called unstable obstacle problem. Elliptic theory and the Sobolev embedding theorem imply that any weak solution belongs to $C^{1, \alpha}$ for any $0<\alpha<1$. It turns out that this is the best one can hope for: there exists a solution which fails to be in $C^{1,1}$ [3]. Hence, if there is a jump at the origin, $C^{1,1}$ regularity can hold only if the jump is positive and this gives rise to:

Assumption B $g_{1}(x, 0)-g_{2}(x, 0) \geq \sigma_{0}, x \in B_{1}$ for some $\sigma_{0}>0$.

The free boundary $\Gamma=\partial\{u \neq 0\}$ consists of two parts: $\Gamma^{0}=\Gamma \cap\{\nabla u=0\}$ and $\Gamma^{1}=\Gamma \cap\{\nabla u \neq 0\}$. The main difficulty in proving $C^{1,1}$ regularity is the analysis of points where the gradient of the function vanishes. In this direction we establish the following result.

Theorem 1.2 Suppose $g_{1}, g_{2}$ satisfy $A$ and $B$. Then if $u$ is a solution of (1), $\|u\|_{C^{1,1}(K)}<\infty$ for any $K \Subset B_{1 / 2}(0) \backslash \overline{\Gamma^{1}}$.

At points where the gradient does not vanish, the implicit function theorem yields that the free boundary is locally a $C^{1, \alpha}$ graph for any $0<\alpha<1$. The solution $u$ changes 
sign across the free boundary, hence it locally solves the equation $\Delta u=g_{1}(x, u)$ on the side where it is positive and $\Delta u=g_{2}(x, u)$ on the side where it is negative. If the coefficients $g_{i}$ are regular enough to provide $C^{1,1}$ solutions up to the boundary-this is encoded in Assumption $\mathrm{C}$ - then we obtain full $C^{1,1}$ regularity.

Assumption C For any $M>0$ there exist $\theta_{0}\left(M,\left\|g_{1}\right\|_{\infty},\left\|g_{2}\right\|_{\infty}, n\right)>0$ and $C_{3}\left(M,\left\|g_{1}\right\|_{\infty},\left\|g_{2}\right\|_{\infty}, n\right)>0$ such that for all $z \in B_{1 / 2}$ any solution of

$$
\left\{\begin{array}{l}
\Delta v=g_{1}(x, v) \chi_{\{v>0\}}+g_{2}(x, v) \chi_{\{v<0\}}, x \in B_{1 / 2}(z) \\
|v(x)| \leq M, x \in B_{1 / 2}(z) \\
v(z)=0,0<|\nabla v(z)| \leq \theta_{0}
\end{array}\right.
$$

admits a bound

$$
\left\|D^{2} v\right\|_{L^{\infty}\left(B_{|\nabla v(z)| / \theta_{0}}(z)\right)} \leq C_{3} .
$$

Remark 1 A sufficient condition which ensures $\mathrm{C}$ is that $g_{i}$ are Hölder continuous, see [12, Proposition 2.6] and [1, Theorem 9.3]. The idea being that at such points, the set $\{u=0\}$ is locally $C^{1, \alpha}$ (via the implicit function theorem) and one may thereby reduce the problem to a classical PDE for which up to the boundary estimates are known.

Theorem 1.3 Suppose $g_{1}, g_{2}$ satisfy $A, B$ and $C$. Let $u$ be a solution of (1) and $0 \in \Gamma^{0}$. Then $u \in C^{1,1}\left(B_{\rho_{0}}(0)\right)$, for some $\rho_{0}>0$.

Equation (1) with right-hand side of the form (2) is a generalization of the wellstudied two-phase membrane problem, where $g_{i}(x, u)=\lambda_{i}(x), i=1,2$. The $C^{1,1}$ regularity in the case when $\lambda_{1} \geq 0, \lambda_{2} \leq 0$ are two constants satisfying $\mathrm{B}$ was obtained by Uraltseva [16] via the ACF monotonicity formula. Moreover, Shahgholian proved this result for Lipschitz coefficients which satisfy B [14, Example 2]. If the coefficients are Hölder continuous, the ACF method does not directly apply and under the stronger assumption that inf $\lambda_{1}>0$ and inf $-\lambda_{2}>0$, Edquist, Lindgren, Shahgholian [12] obtained the $C^{1,1}$ regularity via an analysis of blow-up limits and a classification of global solutions (see also [12, Remark 1.3]). Theorem 1.3 improves and extends this result.

The difficulty in the case when $g_{i}$ depend also on $u$ is that if $v:=u+L$ for some linear function $L$, then $v$ is no longer a solution to the same equation, so one has to get around the lack of linear invariance. Our technique exploits that linear perturbations do not affect certain $L^{2}$ projections.

The proof of Theorem 1.3 does not rely on classical monotonicity formulas or classification of global solutions. Rather, our method is based on an identity which provides monotonicity in $r$ of the square of the $L^{2}$ norm of the projection of $u$ onto the space of second order homogeneous harmonic polynomials on the sphere of radius $r$.

Theorems 1.2 and 1.3 deal with the case when $f$ has a jump discontinuity. If $f$ has a removable discontinuity, (1) has the structure

$$
\Delta u=g(x, u) \chi\{u \neq 0\} .
$$


In this case, one may merge some observations in the proofs of the previous results with the method in [2] and prove the following theorem.

Theorem 1.4 If $g$ satisfies Assumption A, then every solution of (3) is in $C^{1,1}\left(B_{1 / 2}\right)$.

Theorems 1.1-1.4 provide a comprehensive theory for the general semilinear Poisson equation where the free boundary theory is encoded in the regularity assumption of $f$ in the $u$-variable.

\section{Technical tools}

Throughout the text, the right-hand side of (1) is assumed to be bounded. Moreover, $\mathcal{P}_{2}$ denotes the space of second order homogeneous harmonic polynomials. A useful elementary fact is that all norms on $\mathcal{P}_{2}$ are equivalent.

Lemma 2.1 The space $\mathcal{P}_{2}$ is a finite dimensional linear space. Consequently, all norms on $\mathcal{P}_{2}$ are equivalent.

For $u \in W^{2,2}\left(B_{1}\right), y \in B_{1}$ and $r \in\left(0, \operatorname{dist}\left(y, \partial B_{1}\right)\right), \Pi_{y}(u, r)$ is defined to be the $L^{2}$ projection operator on $\mathcal{P}_{2}$ given by

$$
\inf _{h \in \mathcal{P}_{2}} \int_{B_{1}}\left|D^{2} \frac{u(r x+y)}{r^{2}}-D^{2} h\right|^{2} d x=\int_{B_{1}}\left|D^{2} \frac{u(r x+y)}{r^{2}}-D^{2} \Pi_{y}(u, r)\right|^{2} d x .
$$

Calderon-Zygmund theory yields the following useful inequality for re-scalings of weak solutions of (1).

Lemma 2.2 Let $u$ solve (1), $y \in B_{1 / 2}$, and $r \leq 1 / 4$. Then for

$$
\tilde{u}_{r}(x)=\frac{u(r x+y)-r x \cdot \nabla u(y)-u(y)}{r^{2}}
$$

it follows that for $1 \leq p<\infty$ and $0<\alpha<1$,

$$
\left\|\tilde{u}_{r}-\Pi_{y}(u, r)\right\|_{W^{2, p}\left(B_{1}\right)} \leq C\left(n,\|f\|_{L^{\infty}\left(B_{1} \times \mathbb{R}\right)},\|u\|_{L^{\infty}\left(B_{1}\right)}, p\right),
$$

and

$$
\left\|\tilde{u}_{r}-\Pi_{y}(u, r)\right\|_{C^{1, \alpha}\left(B_{1}\right)} \leq C\left(n,\|f\|_{L^{\infty}\left(B_{1} \times \mathbb{R}\right)},\|u\|_{L^{\infty}\left(B_{1}\right)}, \alpha\right) .
$$

Proof By Calderon-Zygmund theory (e.g. [2, Theorem 2.2]),

$$
\left\|D^{2} u\right\|_{B M O\left(B_{1 / 2}\right)} \leq C
$$

in particular,

$$
\int_{B_{3 / 2}}\left|D^{2} \tilde{u}_{r}-\overline{D^{2} \tilde{u}_{r}}\right|^{2} \leq C
$$


where $\overline{D^{2} \tilde{u}_{r}}$ is the average of $D^{2} \tilde{u}_{r}$ on $B_{3 / 2}$. Now let

$$
a=a(f, r, y)=f_{B_{3 / 2}} f(r x+y, u(r x+y)) d x
$$

and note that this quantity is uniformly controlled by $\|f\|_{L^{\infty}}\left(B_{1} \times \mathbb{R}\right)$; this fact, and the definition of $\Pi$ yields (note: $\operatorname{trace}\left(\overline{D^{2} u}-\frac{a}{n} I d\right)=0$ ),

$$
\int_{B_{3 / 2}}\left|D^{2}\left(\tilde{u}_{r}-\Pi_{0}\left(\tilde{u}_{r}, 3 / 2\right)\right)\right|^{2} \leq \int_{B_{3 / 2}}\left|D^{2} \tilde{u}_{r}-\left(\overline{D^{2} u}-\frac{a}{n} I d\right)\right|^{2} \leq C_{1} .
$$

Two applications of Poincaré's inequality together with the above estimate implies

$$
\left\|\tilde{u}_{r}-\Pi_{y}(u, r)-\overline{\nabla \tilde{u}_{r}} \cdot x-\overline{\tilde{u}_{r}}\right\|_{W^{2,2}\left(B_{3 / 2}\right)} \leq C_{2},
$$

where the averages are taken over $B_{3 / 2}$. Elliptic theory (e.g. [7, Theorem 9.1]) yields that for any $1 \leq p<\infty$,

$$
\left\|\tilde{u}_{r}-\Pi_{y}(u, r)-\overline{\nabla \tilde{u}_{r}} \cdot x-\overline{\tilde{u}}_{r}\right\|_{W^{2, p}\left(B_{3 / 2}\right)} \leq C_{3} .
$$

Let $\phi:=\tilde{u}_{r}-\overline{\nabla \tilde{u}_{r}} \cdot x-\overline{\tilde{u}}_{r}$. We have that $\phi(0)=-\overline{\tilde{u}}_{r}$ and $\nabla \phi(0)=-\overline{\nabla \tilde{u}_{r}}$; however, by the Sobolev embedding theorem, $\phi$ is $C^{1, \alpha}$ and thus

$$
|\phi(0)|+|\nabla \phi(0)| \leq C_{4}
$$

completing the proof of the $W^{2, p}$ estimate. The $C^{1, \alpha}$ estimate likewise follows from the Sobolev embedding theorem.

Our analysis requires several additional simple technical lemmas involving the projection operator.

Lemma 2.3 For any $u \in W^{2,2}\left(B_{1}\right)$ and $s \in[1 / 2,1]$,

$$
\left\|\Pi_{0}(u, s)-\Pi_{0}(u, 1)\right\|_{L^{2}\left(B_{1}\right)} \leq C\|\Delta u\|_{L^{2}\left(B_{1}\right)},
$$

and

$$
\left\|\Pi_{0}(u, s)-\Pi_{0}(u, 1)\right\|_{L^{\infty}\left(B_{1}\right)} \leq C\|\Delta u\|_{L^{2}\left(B_{1}\right)},
$$

for some constant $C=C(n)$.

Proof Let $f=\Delta u$ and $v$ be the Newtonian potential of $f$, i.e.

$$
v(x)=\frac{1}{n(n-2) \omega_{n}} \int_{\mathbb{R}^{n}} \frac{f(y) \chi_{B_{1}}(y)}{|x-y|^{n-2}} d x
$$


where $\omega_{n}$ is the volume of the unit ball in $\mathbb{R}^{n}$. Since $u-v$ is harmonic,

$$
\Pi_{0}(u-v, s)=\Pi_{0}(u-v, 1)
$$

therefore

$$
\Pi_{0}(u, s)-\Pi_{0}(u, 1)=\Pi_{0}(v, s)-\Pi_{0}(v, 1) .
$$

Invoking bounds on the projection (e.g. [2, Lemma 3.2]) and Calderon-Zygmund theory (e.g. [2, Theorem 2.2]), it follows that

$$
\begin{aligned}
\left\|\Pi_{0}(u, s)-\Pi_{0}(u, 1)\right\|_{L^{2}\left(B_{1}\right)} & =\left\|\Pi_{0}(v, s)-\Pi_{0}(v, 1)\right\|_{L^{2}\left(B_{1}\right)} \\
& \leq C\|\Delta v\|_{L^{2}\left(B_{1}\right)}=C\|\Delta u\|_{L^{2}\left(B_{1}\right)} .
\end{aligned}
$$

The $L^{\infty}$ bound follows from the equivalence of the norms in the space $\mathcal{P}_{2}$.

Lemma 2.4 Let $u$ solve (1). Then for all $0<r \leq 1 / 4, s \in[1 / 2,1]$ and $y \in B_{1 / 2}$,

$$
\sup _{B_{1}}\left|\Pi_{y}(u, r s)-\Pi_{y}(u, r)\right| \leq C,
$$

and

$$
\sup _{B_{1}}\left|\Pi_{y}(u, r)\right| \leq C \log (1 / r)
$$

for some constant $C=C\left(n,\|f\|_{L^{\infty}\left(B_{1} \times \mathbb{R}\right)},\|u\|_{L^{\infty}\left(B_{1}\right)}\right)$.

Proof Note that

$$
\Pi_{y}(u, r s)-\Pi_{y}(u, r)=\Pi_{0}\left(\tilde{u}_{r}, s\right)-\Pi_{0}\left(\tilde{u}_{r}, 1\right),
$$

where

$$
\tilde{u}_{r}(x)=\frac{u(r x+y)-r x \cdot \nabla u(y)-u(y)}{r^{2}}
$$

as before. From Lemma 2.3 we have that

$$
\left\|\Pi_{0}\left(\tilde{u}_{r}, s\right)-\Pi_{0}\left(\tilde{u}_{r}, 1\right)\right\|_{L^{\infty}\left(B_{1} \times \mathbb{R}\right)} \leq C\left\|\Delta \tilde{u}_{r}\right\|_{L^{2}\left(B_{1}\right)} \leq C\|f\|_{L^{\infty}\left(B_{1}\right)} .
$$

As for the second inequality in the statement of the lemma let $r_{0}=1 / 4$ and $s \in$ $[1 / 2,1]$. Then we have that 


$$
\begin{aligned}
\sup _{B_{1}}\left|\Pi_{y}\left(u, s r_{0} / 2^{j}\right)\right| \leq & \sup _{B_{1}}\left|\Pi_{y}\left(u, s r_{0} / 2^{j}\right)-\Pi_{y}\left(u, r_{0} / 2^{j}\right)\right| \\
& +\sum_{k=0}^{j-1} \sup _{B_{1}} \mid \Pi_{y}\left(u, r / 2^{k+1}\right)-\Pi_{y}\left(u, r / 2^{k}\right) \\
& +\sup _{B_{1}}\left|\Pi_{y}\left(u, r_{0}\right)\right| \leq C j \leq C \log \left(\frac{2^{j}}{s r_{0}}\right),
\end{aligned}
$$

for all

The previous tools imply a growth estimate on weak solutions solution of (1).

Lemma 2.5 Let $u$ solve (1). Then for $y \in B_{1 / 2}$ and $r>0$ small enough,

$$
\sup _{B_{r}(y)}|u(x)-u(y)-(x-y) \nabla u(y)| \leq C r^{2} \log (1 / r) .
$$

Proof Let

$$
\tilde{u}_{r}=\frac{u(r x+y)-r x \cdot \nabla u(y)-u(y)}{r^{2}} .
$$

The assertion of the Lemma is equivalent to the estimate

$$
\left\|\tilde{u}_{r}\right\|_{L^{\infty}\left(B_{1}\right)} \leq C \log (1 / r)
$$

for $r$ small enough. Lemma 2.4 and the $C^{1, \alpha}$ estimates of Lemma 2.2 imply

$$
\begin{aligned}
\left\|\tilde{u}_{r}\right\|_{L^{\infty}\left(B_{1}\right)} & \leq\left\|\tilde{u}_{r}-\Pi_{y}(u, r)\right\|_{L^{\infty}\left(B_{1}\right)}+\left\|\Pi_{y}(u, r)\right\|_{L^{\infty}\left(B_{1}\right)} \\
& \leq C+C \log (1 / r) \leq C \log (1 / r),
\end{aligned}
$$

provided $r$ is small enough.

Next lemma relates the boundedness of the projection operator and the boundedness of second derivatives of weak solutions of (1).

Lemma 2.6 Let $u$ be a solution to (1). If for each $y \in B_{1 / 2}$ there is a sequence $r_{j}(y) \rightarrow 0^{+}$as $j \rightarrow \infty$ such that

$$
M:=\sup _{y \in B_{1 / 2}} \sup _{j \in \mathbb{N}}\left\|D^{2} \Pi_{y}\left(u, r_{j}(y)\right)\right\|_{L^{\infty}\left(B_{1 / 2}\right)}<\infty,
$$

then

$$
\left|D^{2} u\right| \leq C \text { a.e. in } B_{1 / 2}
$$

for some constant $C=C\left(M, n,\|f\|_{L^{\infty}\left(B_{1} \times \mathbb{R}\right)},\|u\|_{L^{\infty}\left(B_{1}\right)}\right)>0$. 
Proof Let $y \in B_{1 / 2}$ be a Lebesgue point for $D^{2} u$ and $r_{j}=r_{j}(y) \rightarrow 0^{+}$as $j \rightarrow \infty$. Then by utilizing Lemma 2.2,

$$
\begin{aligned}
\left|D^{2} u(y)\right| & =\lim _{j \rightarrow \infty} f_{B_{r_{j}}(y)}\left|D^{2} u(z)\right| d z \\
& \leq \limsup _{j \rightarrow \infty} f_{B_{r_{j}}(y)}\left|D^{2} u(z)-D^{2} \Pi_{y}\left(u, r_{j}\right)\right| d z+M \\
& \leq C .
\end{aligned}
$$

Since a.e. $z \in B_{1 / 2}$ is a Lebesgue point for $D^{2} u$, the proof is complete.

Next, we introduce another projection that we need for our analysis. Define $Q_{y}(u, r)$ to be the minimizer of

$$
\inf _{q \in \mathcal{P}_{2}} \int_{\partial B_{1}}\left|\frac{u(r x+y)}{r^{2}}-q(x)\right|^{2} d \mathcal{H}^{n-1} .
$$

The following lemma records the basic properties enjoyed by this projection, cf. [2, Lemma 3.2].

Lemma 2.7 (i) $Q_{y}(\cdot, r)$ is linear;

(ii) if $u$ is harmonic $Q_{y}(u, s)=Q_{y}(u, r)$ for all $s<r$;

(iii) if $u$ is a linear function then $Q_{y}(u, r)=0$;

(iv) if $u$ is a second order homogeneous polynomial then $Q_{y}(u, r)=u$;

(v) $\left\|Q_{0}(u, s)-Q_{0}(u, 1)\right\|_{L^{2}\left(\partial B_{1}\right)} \leq C_{s}\|\Delta u\|_{L^{2}\left(B_{1}\right)}$, for $0<s<1$;

(vi) $\left\|Q_{0}(u, 1)\right\|_{L^{2}\left(\partial B_{1}\right)} \leq\|u\|_{L^{2}\left(\partial B_{1}\right)}$.

Proof (i) This is evident.

(ii) It suffices to prove $Q_{y}(u, r)=Q_{y}(u, 1)$ for $r<1$. Let

$$
\sigma_{2}=\frac{Q_{y}(u, 1)}{\left\|Q_{y}(u, 1)\right\|_{L^{2}\left(\partial B_{1}\right)}}
$$

and for $i \neq 2$, let $\sigma_{i}$ be an $i$ th degree harmonic polynomial. Then there exist coefficients $a_{i}$ such that

$$
u(x+y)=\sum_{i=0}^{\infty} a_{i} \sigma_{i}(x), \quad x \in \partial B_{1}
$$

in particular, $a_{2}=\left\|Q_{y}(u, 1)\right\|_{L^{2}\left(\partial B_{1}\right)}$. Let

$$
v(x)=\sum_{i=0}^{\infty} a_{i} \sigma_{i}(x), \quad x \in B_{1} .
$$


Then $v$ is a harmonic and $u(x+y)=v(x)$ for $x \in \partial B_{1}$. Hence, we have that $u(x+y)=v(x)$ for $x \in B_{1}$ and in particular

$$
u(x+y)=\sum_{i=0}^{\infty} a_{i} \sigma_{i}(x), \quad x \in B_{1} .
$$

Therefore

$$
\frac{u(r x+y)}{r^{2}}=\sum_{i=0}^{\infty} a_{i} \frac{\sigma_{i}(r x)}{r^{2}}=\sum_{i=0}^{\infty} a_{i} r^{i-2} \sigma_{i}(x), \quad x \in B_{1},
$$

so $Q_{y}(u, r)=a_{2} \sigma_{2}(x)=Q_{y}(u, 1)$.

(iii) and (iv) These are evident.

(v) Similar to Lemma 2.3.

(vi) This follows from the fact that $Q_{0}(u, 1)$ is the $L^{2}$ projection of $u$.

We also employ following simple observation in the subsequent analysis.

Remark 2 If $H$ is a Hilbert space and $E \subset H$ a subspace, then for any $x \in H$ and $e \in E,\langle x, e\rangle=\left\langle\operatorname{Proj}_{E}(x), e\right\rangle$ (since we may write $x=\operatorname{Proj}_{E}(x)+y$, where $\left.y \in E^{\perp}\right)$.

Next we prove some technical results for $Q_{y}(u, r)$ and establish a precise connection between $\Pi_{y}(u, r)$ and $Q_{y}(u, r)$ by showing that the difference is uniformly bounded in $r$.

Lemma 2.8 For $u \in W^{2, p}\left(B_{1}(y)\right)$ with $p>n$ and $r \in(0,1]$,

$$
\frac{d}{d r} Q_{y}(u, r)=\frac{1}{r} Q_{0}(x \cdot \nabla u(x+y)-2 u(x+y), r) .
$$

Proof Firstly,

$$
Q_{y}(u, r)=Q_{0}\left(\frac{u(r x+y)}{r^{2}}, 1\right)
$$

Since $u$ is $C^{1, \alpha}$ if $p>n$ and $Q$ is linear bounded operator, it follows that

$$
\begin{aligned}
\frac{d}{d r} Q_{y}(u, r) & =Q_{0}\left(\frac{d}{d r} \frac{u(r x+y)}{r^{2}}, 1\right)=Q_{0}\left(\frac{r x \cdot \nabla u(r x+y)-2 u(r x+y)}{r^{3}}, 1\right) \\
& =\frac{1}{r} Q_{0}(x \cdot \nabla u(x+y)-2 u(x+y), r) .
\end{aligned}
$$


Lemma 2.9 Let $u \in W^{2, p}\left(B_{1}(y)\right)$ with $p>n$ and $q \in \mathcal{P}_{2}$. Then

$$
\int_{B_{1}} q(x) \Delta u(x+y) d x=\int_{\partial B_{1}} q(x)(x \cdot \nabla u(x+y)-2 u(x+y)) d \mathcal{H}^{n-1} .
$$

Proof Integration by parts implies

$$
\begin{aligned}
\int_{B_{1}} q(x) \Delta u(x+y) d x= & \int_{B_{1}} \Delta q(x) u(x+y) d x+\int_{\partial B_{1}} q(x) \frac{\partial u(x+y)}{\partial n} \\
& -u(x+y) \frac{\partial q(x)}{\partial n} d \mathcal{H}^{n-1}
\end{aligned}
$$

By taking into account that $q$ is a second order homogeneous polynomial it follows that

$$
\frac{\partial q(x)}{\partial n}=2 q(x), \quad x \in \partial B_{1}
$$

Moreover,

$$
\frac{\partial u(x+y)}{\partial n}=x \cdot \nabla u(x+y), \quad x \in \partial B_{1}
$$

Combining these equations yields (4).

Lemma 2.10 Let $u \in W^{2, p}\left(B_{1}(y)\right)$ with $p>n$ and $0<r \leq 1$. Then for every $q \in \mathcal{P}_{2}$,

$$
\int_{\partial B_{1}} q(x) \frac{d}{d r} Q_{y}(u, r)(x) d \mathcal{H}^{n-1}=\frac{1}{r} \int_{B_{1}} q(x) \Delta u(r x+y) d x .
$$

Proof Let $\tilde{u}_{r}(x)=u(r x+y) / r^{2}$. From Lemmas 2.8, 2.9, and the fact that $Q_{0}(\cdot, 1)$ is the projection onto the space of homogeneous harmonic polynomials of degree two, we obtain

$$
\begin{array}{rl}
\int_{\partial B_{1}} & q(x) \frac{d}{d r} Q_{y}(u, r)(x) d \mathcal{H}^{n-1} \\
= & \frac{1}{r} \int_{\partial B_{1}} q(x) Q_{0}\left(\frac{r x \cdot \nabla u(r x+y)-2 u(r x+y)}{r^{2}}, 1\right) d \mathcal{H}^{n-1} \\
= & \frac{1}{r} \int_{\partial B_{1}} q(x) Q_{0}\left(x \cdot \nabla \tilde{u}_{r}(x)-2 \tilde{u}_{r}(x), 1\right) d \mathcal{H}^{n-1}
\end{array}
$$




$$
\begin{aligned}
& =\frac{1}{r} \int_{\partial B_{1}} q(x)\left(x \cdot \nabla \tilde{u}_{r}(x)-2 \tilde{u}_{r}(x)\right) d \mathcal{H}^{n-1} \\
& =\frac{1}{r} \int_{B_{1}} q(x) \Delta \tilde{u}_{r}(x) d x=\frac{1}{r} \int_{B_{1}} q(x) \Delta u(r x+y) d x,
\end{aligned}
$$

(the third equality follows from Remark 2).

Lemma 2.11 For $u \in W^{2, p}\left(B_{1}(y)\right)$ with $p>n$ and $0<r \leq 1$,

$$
\frac{d}{d r} \int_{\partial B_{1}} Q_{y}^{2}(u, r) d \mathcal{H}^{n-1}=\frac{2}{r} \int_{B_{1}} Q_{y}(u, r) \Delta u(r x+y) d x .
$$

Proof By Lemmas 2.8, 2.10 we get

$$
\begin{aligned}
\frac{d}{d r} \int_{\partial B_{1}} Q_{y}^{2}(u, r) d \mathcal{H}^{n-1} & =2 \int_{\partial B_{1}} Q_{y}(u, r) \frac{d}{d r} Q_{y}(u, r) d \mathcal{H}^{n-1} \\
& =\frac{2}{r} \int_{B_{1}} Q_{y}(u, r) \Delta u(r x+y) d x .
\end{aligned}
$$

Lemma 2.12 Let $f \in L^{\infty}\left(B_{1}\right)$, $u$ be a solution of (1) and $y \in B_{1 / 2}$. For $0<r<1 / 2$ consider

$$
\begin{aligned}
u_{r}(x) & :=\frac{u(r x+y)-r x \cdot \nabla u(y)-u(y)}{r^{2}}-\Pi_{y}(u, r), \\
v_{r}(x) & :=\frac{u(r x+y)-r x \cdot \nabla u(y)-u(y)}{r^{2}}-Q_{y}(u, r) .
\end{aligned}
$$

Then

(i) $u_{r}-v_{r}$ is bounded in $C^{\infty}$, uniformly in $r$;

(ii) the family $\left\{v_{r}\right\}$ is bounded in $C^{1, \alpha}\left(B_{1}\right) \cap W^{2, p}\left(B_{1}\right)$, for every $0<\alpha<1$ and $p>1$.

Proof (i) For each $r$, the difference $u_{r}-v_{r}=Q_{y}(u, r)-\Pi_{y}(u, r)$ is a second order harmonic polynomial. Therefore, it suffices to show that the $L^{\infty}$ norm of that difference admits a bound independent of $r$. Note that

$$
\begin{aligned}
u_{r}-v_{r} & =Q_{y}(u, r)-\Pi_{y}(u, r) \\
& =Q_{0}\left(\frac{u(r x+y)-r x \cdot \nabla u(y)-u(y)}{r^{2}}-\Pi_{y}(u, r), 1\right)=Q_{0}\left(u_{r}, 1\right) .
\end{aligned}
$$


Hence, by Lemma 2.2,

$$
\sup _{r} \sup _{B_{1}}\left|Q_{0}\left(u_{r}, 1\right)\right| \leq C \sup _{r} \sup _{B_{1}}\left|u_{r}\right|<\infty
$$

(ii) Lemma 2.2 implies that $\left\{u_{r}\right\}_{r>0}$ is bounded in $C^{1, \alpha}\left(B_{1}\right) \cap W^{2, p}\left(B_{1}\right)$ for every $\alpha<1$ and $p>1$. Hence, the result follows from (i).

\section{$3 C^{1,1}$ regularity: general case}

In this section we utilize the previous technical tools and prove $C^{1,1}$ regularity provided that $f=f(x, t)$ satisfies Assumption A:

\section{Assumption A (i)}

$$
\left|f\left(x, t_{2}\right)-f\left(x, t_{1}\right)\right| \leq \omega\left(\left|t_{2}-t_{1}\right|\right)
$$

and

$$
\int_{0}^{\epsilon} \frac{\omega(t)}{t} d t<\infty
$$

for some $\epsilon>0$;

(ii) The Newtonian potential of $x \mapsto f(x, t)$ is $C^{1,1}$ locally uniformly in $t$ : for $v_{t}:=f(\cdot, t) * N$ where $N$ is the Newtonian potential,

$$
\sup _{a \leq t \leq b}\left\|D^{2} v_{t}\right\|_{L^{\infty}\left(B_{1}\right)}<\infty, \quad \text { for all } a, b \in \mathbb{R} .
$$

Proof of Theorem 1.1 Let $y \in B_{1 / 2}$ and $v=v_{u(y)}=f(x, u(y)) * N$. Note that if

$$
u_{r}(x)=\frac{u(r x+y)-r x \cdot \nabla u(y)-u(y)}{r^{2}}-\Pi_{y}(u, r),
$$

then

$$
\Pi_{y}(u, r / 2)-\Pi_{y}(u, r)=\Pi_{y}\left(u_{r}, 1 / 2\right)-\Pi_{y}\left(u_{r}, 1\right)=\Pi_{y}\left(u_{r}, 1 / 2\right) .
$$

Using this identity, Lemmas 2.3 and 2.5

$$
\begin{aligned}
& \left\|\Pi_{y}(u, r / 2)-\Pi_{y}(u, r)-\Pi_{y}(v, r / 2)+\Pi_{y}(v, r)\right\|_{L^{\infty}\left(B_{1}\right)} \\
& \quad=\left\|\Pi_{y}\left(u_{r}, 1 / 2\right)-\Pi_{y}\left(v_{r}, 1 / 2\right)-\Pi_{y}\left(u_{r}, 1\right)+\Pi_{y}\left(v_{r}, 1\right)\right\|_{L^{\infty}\left(B_{1}\right)} \\
& =\left\|\Pi_{y}\left(u_{r}-v_{r}, 1 / 2\right)-\Pi_{y}\left(u_{r}-v_{r}, 1\right)\right\|_{L^{\infty}\left(B_{1}\right)} \\
& \leq C\left\|\Delta u_{r}-\Delta v_{r}\right\|_{L^{2}\left(B_{1}\right)}
\end{aligned}
$$




$$
\begin{aligned}
& =\|f(r x+y, u(r x+y))-f(r x+y, u(y))\|_{L^{2}\left(B_{1}\right)} \\
& \leq C \omega\left(\sup _{B_{r}(y)}|u(x)-u(y)|\right) \leq C \omega\left(c\left(r+r^{2} \log \frac{1}{r}\right)\right) \leq C \omega(c r),
\end{aligned}
$$

for $r>0$ sufficiently small $\left(|\nabla u(y)|\right.$ is controlled by $\left.\|u\|_{W^{2, p}\left(B_{1}\right)}\right)$. Hence, for $r_{0}>0$ small enough and $y \in B_{1 / 2}$ we have

$$
\begin{aligned}
& \left\|\Pi_{y}\left(u, r_{0} / 2^{j}\right)-\Pi_{y}\left(u, r_{0}\right)\right\|_{L^{\infty}\left(B_{1}\right)} \\
& \leq\left\|\sum_{k=1}^{j} \Pi_{y}\left(v, r_{0} / 2^{k}\right)-\Pi_{y}\left(v, r_{0} / 2^{k-1}\right)\right\|_{L^{\infty}\left(B_{1}\right)} \\
& \quad+\sum_{k=1}^{j}\left\|\Pi_{y}\left(u, r_{0} / 2^{k}\right)-\Pi_{y}\left(u, r_{0} / 2^{k-1}\right)-\Pi_{y}\left(v, r_{0} / 2^{k}\right)+\Pi_{y}\left(v, r_{0} / 2^{k-1}\right)\right\|_{L^{\infty}\left(B_{1}\right)} \\
& \leq C\left\|D^{2} v_{u(y)}\right\| L^{\infty}\left(B_{1}\right)+C \sum_{k=1}^{\infty} \omega\left(\frac{c r}{2^{k-1}}\right) \leq \tilde{C}\left(\left\|D^{2} v_{u(y)}\right\|_{L^{\infty}\left(B_{1}\right)}+1\right) \\
& \quad \leq \tilde{C}\left(\sup _{|s| \leq \sup |u|}\left\|D^{2} v_{s}\right\|_{L^{\infty}\left(B_{1}\right)}+1\right) .
\end{aligned}
$$

Thus

$$
\left\|\Pi_{y}\left(u, r_{0} / 2^{j}\right)\right\|_{L^{\infty}\left(B_{1}\right)} \leq\left\|\Pi_{y}\left(u, r_{0}\right)\right\|_{L^{\infty}\left(B_{1}\right)}+\tilde{C}\left(\left\|D^{2} v_{u(y)}\right\|_{L^{\infty}\left(B_{1}\right)}+1\right) .
$$

We conclude via Lemmas 2.4 and 2.6.

Remark 3 To generate examples, consider $f(x, t)=\phi(x) \psi(t)$. If $\phi \in L^{\infty}$ and $\psi$ is Dini, then $f$ satisfies condition (i). If $\phi * N$ is $C^{1,1}$ and $\psi$ is locally bounded, then $f$ satisfies (ii). Thus if $\phi * N$ is $C^{1,1}$ and $\psi$ is Dini, then $f$ satisfies both conditions. In particular, $f$ may be strictly weaker than Dini in the $x$-variable.

Remark 4 The projection $Q_{y}$ has similar properties to $\Pi_{y}$. Consequently, if $f$ satisfies Assumption A, (5) holds for $\Pi_{y}$ replaced by $Q_{y}$,

$$
\left\|Q_{y}\left(u, r_{0} / 2^{j}\right)\right\|_{L^{\infty}\left(B_{1}\right)} \leq\left\|Q_{y}\left(u, r_{0}\right)\right\|_{L^{\infty}\left(B_{1}\right)}+\tilde{C}\left(\left\|D^{2} v_{u(y)}\right\|_{L^{\infty}\left(B_{1}\right)}+1\right) .
$$

\section{$4 C^{1,1}$ regularity: discontinuous case}

The goal of this section is to investigate the optimal regularity for solutions of (1) with $f$ having a jump discontinuity in the $t$-variable. This case may be viewed as a free boundary problem. The idea is to employ again an $L^{2}$ projection operator. 


\subsection{Two-phase obstacle problem}

Suppose $f=f(x, u)$ has the form

$$
f(x, u)=g_{1}(x, u) \chi_{\{u>0\}}+g_{2}(x, u) \chi_{\{u<0\}},
$$

where $g_{1}, g_{2}$ are continuous. We recall from the introduction that if $f$ has a jump in $u$ at the origin, then we assume it to be a positive jump:

Assumption B $g_{1}(x, 0)-g_{2}(x, 0) \geq \sigma_{0}, x \in B_{1}$ for some $\sigma_{0}>0$.

Remark 5 In the unstable obstacle problem, i.e. $g_{1}=-1, g_{2}=0$, there exists a solution which is $C^{1, \alpha}$ for any $\alpha \in(0,1)$ but not $C^{1,1}$.

Let $\Gamma^{0}:=\Gamma \cap\{|\nabla u|=u=0\}$ and $\Gamma^{1}:=\Gamma \cap\{|\nabla u| \neq 0\}$. Our main result provides optimal growth away from points with sufficiently small gradients.

Theorem 4.1 Suppose $g_{1}, g_{2} \in C^{0}$ satisfy $B$. Then for all constants $\theta, M>0$ there exist $r_{0}\left(\theta, M,\left\|g_{1}\right\|_{\infty},\left\|g_{2}\right\|_{\infty}, n\right)>0$ and $C_{0}\left(\theta, M,\left\|g_{1}\right\|_{\infty},\left\|g_{2}\right\|_{\infty}, n\right)>0$ such that for any solution of (1) with $\|u\|_{L^{\infty}\left(B_{1}\right)} \leq M$

$$
\left\|Q_{y}(u, r)\right\|_{L^{2}\left(\partial B_{1}(0)\right)} \leq C_{0},
$$

for all $y, r$ such that $r \leq r_{0}$ and $y \in B_{1 / 2} \cap \Gamma \cap\{|\nabla u(y)|<\theta r\}$. Consequently, for the same choice of $r$ and $y$ we have that

$$
\sup _{x \in B_{r}}|u(x+y)-x \cdot \nabla u(y)| \leq C_{1} r^{2}
$$

for some constant $C_{1}\left(\theta, M,\left\|g_{1}\right\|_{\infty},\left\|g_{2}\right\|_{\infty}, n\right)>0$.

The proof of the theorem is carried out in several steps. A crucial ingredient is the following monotonicity result.

Lemma 4.2 Suppose $g_{1}, g_{2} \in C^{0}$ satisfy $B$. Then for all constants $\theta, M>0$ there exist $\kappa_{0}\left(\theta, M,\left\|g_{1}\right\|_{\infty},\left\|g_{2}\right\|_{\infty}, n\right)>0$ and $r_{0}\left(\theta, M,\left\|g_{1}\right\|_{\infty},\left\|g_{2}\right\|_{\infty}, n\right)>0$ such that for any solution $u$ of (1) with $\|u\|_{L^{\infty}\left(B_{1}\right)} \leq M$ if

$$
\left\|Q_{y}(u, r)\right\|_{L^{2}\left(\partial B_{1}\right)} \geq \kappa_{0},
$$

for some $0<r<r_{0}$ and $y \in B_{1 / 2} \cap \Gamma \cap\{|\nabla u(y)|<\theta r\}$, then

$$
\frac{d}{d r} \int_{\partial B_{1}} Q_{y}^{2}(u, r) d \mathcal{H}^{n-1}>0 .
$$


Proof If the conclusion is not true, then there exist radii $r_{k} \rightarrow 0$, solutions $u_{k}$ and points $y_{k} \in B_{1 / 2} \cap \Gamma_{k} \cap\left\{\left|\nabla u_{k}\left(y_{k}\right)\right|<\theta r_{k}\right\}$ such that $\left\|u_{k}\right\|_{L^{\infty}\left(B_{1}\right)} \leq M$, and $\left\|Q_{y_{k}}\left(u_{k}, r_{k}\right)\right\|_{L^{2}\left(\partial B_{1}\right)} \rightarrow \infty$, and

$$
\left.\frac{d}{d r} \int_{\partial B_{1}} Q_{y_{k}}^{2}\left(u_{k}, r\right) d \mathcal{H}^{n-1}\right|_{r=r_{k}} \leq 0
$$

Let

$$
T_{k}:=\left\|Q_{y_{k}}\left(u_{k}, r_{k}\right)\right\|_{L^{2}\left(\partial B_{1}\right)},
$$

and consider the sequence

$$
v_{k}(x)=\frac{u_{k}\left(r_{k} x+y_{k}\right)-r_{k} x \cdot \nabla u_{k}\left(y_{k}\right)}{r_{k}^{2}}-Q_{y_{k}}\left(u_{k}, r_{k}\right) \text {. }
$$

Without loss of generality we can assume that $y_{k} \rightarrow y_{0}$ for some $y_{0} \in B_{1 / 2}$. Lemma 2.2 implies the existence of a function $v$ such that up to a subsequence

$v_{k}(x)=\frac{u_{k}\left(r_{k} x+y_{k}\right)-r_{k} x \cdot \nabla u_{k}\left(y_{k}\right)}{r_{k}^{2}}-Q_{y_{k}}\left(u_{k}, r_{k}\right) \rightarrow v$, in $C_{\mathrm{loc}}^{1, \alpha}\left(\mathbb{R}^{n}\right) \cap W_{\mathrm{loc}}^{2, p}\left(\mathbb{R}^{n}\right)$.

By $C^{1, \alpha}$ convergence, $v(0)=|\nabla v(0)|=0$. Moreover, for $q_{k}(x):=Q_{y_{k}}\left(u_{k}, r_{k}\right) / T_{k}$, we can assume that up to a further subsequence, $q_{k} \rightarrow q$ in $C^{\infty}$ for some $q \in \mathcal{P}_{2}$. Note that

$$
\begin{aligned}
\Delta v_{k}(x)= & g_{1}\left(r_{k} x+y_{k}, u_{k}\left(r_{k} x+y_{k}\right)\right) \chi_{\left\{u_{k}\left(r_{k} x+y_{k}\right)>0\right\}} \\
& +g_{2}\left(r_{k} x+y_{k}, u_{k}\left(r_{k} x+y_{k}\right)\right) \chi_{\left\{u_{k}\left(r_{k} x+y_{k}\right)<0\right\}}
\end{aligned}
$$

hence

$$
\Delta v_{k} \rightarrow \Delta v=g_{1}\left(y_{0}, 0\right) \chi_{\{q(x)>0\}}+g_{2}\left(y_{0}, 0\right) \chi_{\{q(x)<0\}} .
$$

By Lemma 2.11,

$$
\begin{aligned}
0 \geq\left.\frac{d}{d r} \int_{\partial B_{1}} Q_{y_{k}}^{2}\left(u_{k}, r\right) d \mathcal{H}^{n-1}\right|_{r=r_{k}} & =\frac{2}{r_{k}} \int_{B_{1}} Q_{y_{k}}\left(u_{k}, r_{k}\right) \Delta u_{k}\left(r_{k} x+y_{k}\right) d x \\
& =\frac{2 T_{k}}{r_{k}} \int_{B_{1}} q_{k}(x) \Delta v_{k}(x) d x .
\end{aligned}
$$

Therefore

$$
\int_{B_{1}} q_{k}(x) \Delta v_{k}(x) d x \leq 0 .
$$


On the other hand

$$
\begin{aligned}
\lim _{k \rightarrow \infty} \int_{B_{1}} q_{k}(x) \Delta v_{k}(x) d x & =\int_{B_{1}} q(x)\left(g_{1}\left(0, y_{0}\right) \chi_{\{q(x)>0\}}+g_{2}\left(0, y_{0}\right) \chi_{\{q(x)<0\}}\right) d x \\
& =\left(g_{1}\left(0, y_{0}\right)-g_{2}\left(0, y_{0}\right)\right) \int_{\{q(x)>0\}} q(x) d x>0,
\end{aligned}
$$

a contradiction.

Proof of Theorem 4.1 Let $\kappa_{0}$ and $r_{0}$ be the constants from Lemma 4.2. Without loss of generality we can assume that $r_{0} \leq 1 / 4$. From Lemmas 2.4 and 2.12 we have that

$$
\left\|Q_{y}\left(u, r_{0}\right)\right\|_{L^{2}\left(\partial B_{1}\right)} \leq C \log \frac{1}{r_{0}},
$$

for all $y \in B_{1 / 2}$, where $C=C\left(M,\left\|g_{1}\right\|_{\infty},\left\|g_{2}\right\|_{\infty}, n\right)$ is a constant. Let

$$
C_{0}=\max \left(\kappa_{0}, 2 C \log \frac{1}{r_{0}}\right) .
$$

We claim that

$$
\left\|Q_{y}(u, r)\right\|_{L^{2}\left(\partial B_{1}\right)} \leq C_{0},
$$

for $r \leq r_{0}$ and $y \in B_{1 / 2} \cap \Gamma \cap\{|\nabla u(y)|<\theta r\}$. Let us fix $y$ such that $|\nabla u(y)| \leq \theta r_{0}$ and consider

$$
T_{y}(r):=\left\|Q_{y}(u, r)\right\|_{L^{2}\left(\partial B_{1}\right)}
$$

as a function of $r$ on the interval $|\nabla u(y)| / \theta \leq r \leq r_{0}$. Let

$$
e:=\inf \left\{r \text { s.t. } T_{y}(r) \leq C_{0}\right\}
$$

By (9) and (10), we have that $T_{y}\left(r_{0}\right) \leq C_{0}$, so $|\nabla u(y)| / \theta \leq e \leq r_{0}$. If $e>|\nabla u(y)| / \theta$ then $T_{y}(e)=C_{0}$ and by Lemma 4.2 we have that $T_{y}^{\prime}(e)>0$, so $T_{y}(r)<C_{0}$ for $e-\varepsilon<r<e$ which contradicts (11).

Therefore, $e=|\nabla u(y)| / \theta$ and $T_{y}(r) \leq C_{0}$ for all $|\nabla u(y)| / \theta \leq r \leq r_{0}$ which proves (7).

Inequality (8) follows from Lemmas 2.2 and 2.12.

Theorem 4.1 implies $C^{1,1}$ regularity away from $\Gamma^{1}$ in the case the coefficients $g_{i}$ are regular enough to provide $C^{1,1}$ solutions away from the free boundary, i.e. Theorem 1.2 .

Remark 6 Note that A is the condition given in Theorem 1.1. If $g_{i}$ only depend on $x$, then this reduces to the assumption that the Newtonian potential of $g_{i}$ is $C^{1,1}$, which is sharp. 
Proof of Theorem 1.2 Suppose A and B hold. We show that for every $\delta>0$ there exists $C_{\delta}>0$ such that for all $y \in B_{1 / 2}(0)$ such that $\operatorname{dist}\left(y, \Gamma^{1}\right) \geq \delta$, there exists $r_{y}>0$ such that

$$
\left\|Q_{y}(u, r)\right\|_{L^{2}\left(\partial B_{1}(0)\right)} \leq C_{\delta}
$$

for $r \leq r_{y}$.

Consequently,

$$
|u(x)-u(y)-\nabla u(y)(x-y)| \leq \tilde{C}_{\delta}|x-y|^{2}
$$

for $|x-y| \leq r_{y}, \quad y \in B_{1 / 2}(0)$ and $\operatorname{dist}\left(y, \Gamma^{1}\right) \geq \delta$; this readily yields the desired result.

Note that (13) follows from (12) via Lemmas 2.2 and 2.12.

Without loss of generality assume that $\delta \leq r_{0}$, where $r_{0}>0$ is the constant from Theorem 4.1. For every $y \in B_{1 / 2}(0)$ consider the ball $B_{\delta / 2}(y)$. Then there are two possibilities.

(i) $B_{\delta / 2}(y) \cap \Gamma^{0}=\emptyset$.

In this case $B_{\delta / 2} \cap \Gamma=\emptyset$, hence $u$ satisfies the equation

$$
\Delta u=g_{i}(x, u)
$$

in $B_{\delta / 2}(y)$ for $i=1$ or $i=2$. Inequality (6) in Remark 4 and Assumption A yield

$$
\left\|Q_{y}(u, r)\right\|_{L^{2}\left(\partial B_{1}(0)\right)} \leq C \log \frac{4}{\delta}+C\left(\left\|D^{2} v_{u(y)}^{i}\right\|_{L^{\infty}\left(B_{1}\right)}+1\right) \leq C_{\delta},
$$

for $r \leq \delta / 4$, where $v_{u(y)}^{i}$ is defined as the solution to $\Delta w(x)=g_{i}(x, u(y))$.

(ii) $B_{\delta / 2}(y) \cap \Gamma^{0} \neq \emptyset$.

Let $w \in \Gamma^{0}$ be such that $d:=|y-w|=\operatorname{dist}\left(y, \Gamma_{0}\right)$. We have that $d \leq \delta / 2$. As in the previous step, (6) and Assumption A yield

$$
\left\|Q_{y}(u, r)\right\|_{L^{2}\left(\partial B_{1}(0)\right)} \leq\left\|Q_{y}(u, d / 2)\right\|_{L^{2}\left(\partial B_{1}(0)\right)}+C\left(\left\|D^{2} v_{u(y)}^{i}\right\|_{\infty}+1\right),
$$

for $r \leq d / 2$. From Theorem 4.1 we have that

$$
\left|u\left(y+\frac{d}{2} z\right)\right| \leq C\left|y+\frac{d}{2} z-w\right|^{2} \leq C d^{2},
$$

for all $|z| \leq 1$ because $d \leq \delta / 2 \leq r_{0}$. On the other hand, by definition,

$$
Q_{y}(u, d / 2)=\operatorname{Proj}_{\mathcal{P}_{2}}\left(\frac{u\left(y+\frac{d}{2} z\right)}{d^{2} / 4}\right),
$$


where $\operatorname{Proj}_{\mathcal{P}_{2}}$ is the $L^{2}\left(\partial B_{1}(0)\right)$ projection on the space $\mathcal{P}_{2}$. Hence, by Lemma 2.7 vi,

$$
\left\|Q_{y}(u, d / 2)\right\|_{L^{2}\left(\partial B_{1}(0)\right)} \leq\left\|\frac{u\left(y+\frac{d}{2} z\right)}{d^{2} / 4}\right\|_{L^{2}\left(\partial B_{1}(0)\right)} \leq C,
$$

which yields

$$
\left\|Q_{y}(u, r)\right\|_{L^{2}\left(\partial B_{1}(0)\right)} \leq C+C\left(\left\|D^{2} v_{u(y)}^{i}\right\|_{\infty}+1\right),
$$

for $r \leq d / 2$.

The proof is now complete.

Lastly we point out that if the coefficients $g_{i}$ are regular enough to provide $C^{1,1}$ solutions at points where the gradient does not vanish, then we obtain full interior $C^{1,1}$ regularity. Recall from the introduction the following assumption:

Assumption C For any $M>0$ there exist $\theta_{0}\left(M,\left\|g_{1}\right\|_{\infty},\left\|g_{2}\right\|_{\infty}, n\right)>0$ and $C_{3}\left(M,\left\|g_{1}\right\|_{\infty},\left\|g_{2}\right\|_{\infty}, n\right)>0$ such that for all $z \in B_{1 / 2}$ any solution of

$$
\left\{\begin{array}{l}
\Delta v=g_{1}(x, v) \chi_{\{v>0\}}+g_{2}(x, v) \chi_{\{v<0\}}, x \in B_{1 / 2}(z) \\
|v(x)| \leq M, x \in B_{1 / 2}(z) \\
v(z)=0,0<|\nabla v(z)| \leq \theta_{0}
\end{array}\right.
$$

admits a bound

$$
\left\|D^{2} v\right\|_{L^{\infty}\left(B_{|\nabla v(z)| / \theta_{0}}(z)\right)} \leq C_{3} .
$$

Theorem 4.1 and C imply Theorem 1.3.

Proof of Theorem 1.3 Our strategy is to consider several cases. The main idea is to note that by Lemmas 2.12 and 2.6 the assertion follows if we show that there exist $\rho_{0}, C>0$ such that for every $y \in B_{\rho_{0}}(0)$ there exists $r_{y}>0$ such that

$$
\left\|Q_{y}(u, r)\right\|_{L^{2}\left(\partial B_{1}(0)\right)} \leq C
$$

for $0<r \leq r_{y}$.

Let $\rho_{0}$ be such that $|\nabla u(y)| \leq \theta_{0}$ for $y \in B_{\rho_{0}}(0)$, where $\theta_{0}$ is the constant from Assumption $C$ (we can do this because $u$ is $C^{1, \alpha}$ and $0 \in \Gamma^{0}$ ). For $y \in B_{\rho_{0}}(0)$ let $d:=\operatorname{dist}(y, \Gamma)$ and let $w \in \Gamma$ be such that $d=|y-w|$.

From Theorem 1.2 we can assume that $2 d<r_{0}$ where $r_{0}$ is the constant in Theorem 4.1. One of the following cases is possible.

(i) $d=0, y \in \Gamma^{0}$.

In this case we have that (14) holds for $r \leq r_{0}$ by Theorem 4.1 (note that $\nabla u(y)=0)$.

(ii) $d=0, y \in \Gamma^{1}$.

Here, the $C^{1,1}$ bound follows directly from Assumption C. 
(iii) $d>0, w \in \Gamma^{0}$. Here, we repeat the argument in case (ii) of the proof of Theorem

1.2 line by line and obtain that (14) is valid for $r \leq d / 2$.

(iv) $d>0, w \in \Gamma^{1}$.

From Theorem 4.1 we have that

$$
|u(\tilde{z}+w)-\tilde{z} \cdot \nabla u(w)| \leq C_{1}|\tilde{z}|^{2}
$$

for $|\nabla u(w)| / \theta_{0} \leq|\tilde{z}| \leq r_{0}$. On the other hand by Assumption $\mathrm{C}$ we obtain that (15) holds for $|\tilde{z}| \leq|\nabla u(w)| / \theta_{0}$. Hence, (15) holds for all $\tilde{z}$ such that $|\tilde{z}| \leq r_{0}$. Thanks to Assumption A, we have via Remark 4 that

$$
\left\|Q_{y}(u, r)\right\|_{L^{2}\left(\partial B_{1}(0)\right)} \leq\left\|Q_{y}(u, d / 2)\right\|_{L^{2}\left(\partial B_{1}(0)\right)}+C\left(\left\|D^{2} v_{u(y)}^{i}\right\|_{L^{\infty}\left(B_{1}\right)}+1\right),
$$

for $r \leq d / 2$.

Furthermore, by the projection's invariance of affine additions

$$
\begin{aligned}
Q_{y}(u, d / 2) & =\operatorname{Proj}_{\mathcal{P}_{2}}\left(\frac{u\left(y+\frac{d}{2} z\right)-\frac{d}{2} z \cdot \nabla u(y)-u(y)}{d^{2} / 4}\right) \\
& =\operatorname{Proj}_{\mathcal{P}_{2}}\left(\frac{u\left(\left(y+\frac{d}{2} z\right)-\left(y+\frac{d}{2} z-w\right) \cdot \nabla u(w)\right.}{d^{2} / 4}\right) .
\end{aligned}
$$

Hence by applying (15) with $\tilde{z}=y+\frac{d}{2} z-w$ we have that

$$
\begin{aligned}
\left\|Q_{y}(u, d / 2)\right\|_{L^{2}\left(\partial B_{1}(0)\right)} & \leq\left\|\frac{u\left(w+\left(y+\frac{d}{2} z-w\right)\right)-\left(y+\frac{d}{2} z-w\right) \cdot \nabla u(w)}{d^{2} / 4}\right\|_{L^{2}\left(\partial B_{1}(0)\right)} \\
& \leq C
\end{aligned}
$$

which combined with (16) yields

$$
\left\|Q_{y}(u, r)\right\|_{L^{2}\left(\partial B_{1}(0)\right)} \leq C+C\left(\left\|D^{2} v_{u(y)}^{i}\right\|_{L^{\infty}\left(B_{1}\right)}+1\right)
$$

for $r \leq d / 2$. Note that $|\tilde{z}| \leq r_{0}$ is guaranteed because we have chosen $d<\frac{r_{0}}{2}$.

The previous analysis applies to the following example.

Example Let $g_{i}(x, u)=\lambda_{i}(x)$ for $i=1,2$, where $\lambda_{i}$ are such that

(i) $\lambda_{1}(x)-\lambda_{2}(x) \geq \sigma_{0}>0$ for all $x \in B_{1}$;

(ii) $\lambda_{1}(x), \lambda_{2}(x)$ are Hölder continuous.

We recall from the introduction that under the stronger assumption $\inf _{B_{1}} \lambda_{1}>0$, $\inf _{B_{1}}-\lambda_{2}>0$, this problem is studied in [12] and the optimal interior $C^{1,1}$ regularity is established. The authors use a different approach based on monotonicity formulas and an analysis of global solutions via a blow-up procedure. 


\subsection{No-sign obstacle problem}

Here we observe that Assumption A implies that the solutions of (3) are in $C^{1,1}\left(B_{1 / 2}\right)$. This theorem was proven in [2] (Theorem 1.2) for the case when $g(x, t)$ depends only on $x$. Under Assumption A, appropriate modifications of the proof in [2] work also for the general case; since the arguments are similar, we provide only a sketch of the proof and highlight the differences.

Sketch of the proof of Theorem 1.4 Let $\tilde{\Gamma}:=\{y$ s.t. $u(y)=|\nabla u(y)|=0\}$. For $r>0$ let $\Lambda_{r}:=\left\{x \in B_{1}\right.$ s.t. $\left.u(r x)=0\right\}$ and $\lambda_{r}:=\left|\Lambda_{r}\right|$.

The proof of Theorem 1.2 in [2] consists of the following ingredients.

- Interior $C^{1,1}$ estimate

- Quadratic growth away from the free boundary

- [2, Proposition 5.1]

Let us recall that the interior $C^{1,1}$ estimate is the inequality

$$
\|u\|_{C^{1,1}\left(B_{d / 2}\right)} \leq C\left(\left\|D^{2} v\right\|_{L^{\infty}\left(B_{d}\right)}+\frac{\|u\|_{L^{\infty}\left(B_{d}\right)}}{d^{2}}\right),
$$

where $\Delta u(x)=g(x)$ for $x \in B_{d}$ and $v$, the Newtonian potential of $g$, is $C^{1,1}$. This estimate is purely a consequence of $g$ having a $C^{1,1}$ Newtonian potential.

Quadratic growth away from the free boundary is a bound

$$
|u(x)| \leq C \operatorname{dist}(x, \tilde{\Gamma})^{2} .
$$

The first observation in [2] is that if $g(x, t)=g(x)$ has a $C^{1,1}$ Newtonian potential, then (17) and (18) yield $C^{1,1}$ regularity for the solution. Indeed, "far" from the free boundary, the solution $u$ solves the equation $\Delta u=g(x)$ and is locally $C^{1,1}$ by assumption. For points close to the free boundary, $u$ solves the same equation but now on a small ball centered at the point of interest and touching the free boundary. At this point one invokes (18) and by (17) obtains that the $C^{1,1}$ bound does not blow up close to the free boundary (see Lemma 4.1 in [2]).

To prove (18), the authors prove in Proposition 5.1 [2] that if the projection $\Pi_{y}(u, r)$ (for some $y \in \tilde{\Gamma}$ ) is large enough then the density $\lambda_{r}$ of the coincidence set diminishes at an exponential rate. On the other hand, if $\lambda_{r}$ diminishes at an exponential rate, $\Pi_{y}(u, r)$ has to be bounded. Consequently, by invoking Lemma 2.2 one obtains (18).

Now let $g$ satisfy A.

- Interior $C^{1,1}$ estimate

In the general case, (17) is replaced by

$$
\left\|Q_{y}(u, s)\right\|_{L^{2}\left(\partial B_{1}(0)\right)} \leq\left\|Q_{y}(u, r)\right\|_{L^{2}\left(\partial B_{1}(0)\right)}+C\left(\left\|D^{2} v_{u(y)}\right\|_{\infty}+1\right),
$$

where $0<s<r<d, \Delta v_{u(y)}=g(x, u(y))$ and $\Delta u=f(x, u)$ in $B_{d}(y)$. Estimate (19) is purely a consequence of Assumption A via Remark 4. 
- [2, Proposition 5.1]

In this proposition, it is shown that there exists $C$ such that if $\Pi_{y}(u, r) \geq C$ then

$$
\lambda_{r / 2}^{1 / 2} \leq \frac{\tilde{C}}{\left\|\Pi_{y}(u, r)\right\|_{L^{\infty}\left(B_{1}\right)}} \lambda_{r}^{1 / 2}
$$

for some $\tilde{C}>0$. The inequality is obtained by the decomposition

$$
\frac{u(r x+y)}{r^{2}}=\Pi_{y}(u, r)+h_{r}+w_{r}
$$

where $h_{r}, w_{r}$ are such that

$$
\begin{cases}\Delta h_{r}=-g(r x+y) \chi_{\Lambda_{r}} & \text { in } B_{1} \\ h_{r}=0 & \text { on } \partial B_{1}\end{cases}
$$

and

$$
\begin{cases}\Delta w_{r}=g(r x+y) & \text { in } B_{1}, \\ w_{r}=\frac{u(r x+y)}{r^{2}}-\Pi_{y}(u, r) & \text { on } \partial B_{1} .\end{cases}
$$

The authors show that

$$
\begin{aligned}
\left\|D^{2} h_{r}\right\|_{L^{2}\left(B_{1 / 2}\right)} & \leq C\|g\|_{L^{\infty}\left\|\chi_{\Lambda_{r}}\right\|_{L^{2}\left(B_{1}\right)},} \\
\left\|D^{2} w_{r}\right\|_{L^{\infty}\left(B_{1 / 2}\right)} & \leq C\left(\|g\|_{L^{\infty}}+\|u\|_{L^{\infty}\left(B_{1}\right)}\right) .
\end{aligned}
$$

In the general case one may consider the decomposition

$$
\frac{u(r x+y)}{r^{2}}=Q_{y}(u, r)+h_{r}+w_{r}+z_{r},
$$

where $h_{r}, w_{r}, z_{r}$ are such that

$$
\begin{cases}\Delta h_{r}=-g(r x+y, 0) \chi_{\Lambda_{r}} & \text { in } B_{1} \\ h_{r}=0 & \text { on } \partial B_{1},\end{cases}
$$

and

$$
\begin{cases}\Delta w_{r}=g(r x+y, 0) & \text { in } B_{1}, \\ w_{r}=\frac{u(r x+y)}{r^{2}}-Q_{y}(u, r) & \text { on } \partial B_{1},\end{cases}
$$

and

$$
\begin{cases}\Delta z_{r}=(g(r x+y, u(r x+y))-g(r x+y, 0)) \chi_{B_{1} \backslash \Lambda_{r}} & \text { in } B_{1}, \\ z_{r}=0 & \text { on } \partial B_{1} .\end{cases}
$$


Evidently, estimates (21) are still valid. Additionally, we have

$$
\left\|D^{2} z_{r}\right\|_{L^{2}\left(B_{1 / 2}\right)} \leq C\left\|\Delta z_{r}\right\|_{L^{2}\left(B_{1}\right)} \leq C \omega\left(r^{2} \log \frac{1}{r}\right)
$$

since $g(x, t)$ is uniformly Dini in $t$.

Combining (21) and (22) and arguing as in [2] one obtains the existence of $C>0$ such that

$$
\lambda_{r / 2}^{1 / 2} \leq \frac{\tilde{C}}{\left\|Q_{y}(u, r)\right\|_{L^{2}\left(\partial B_{1}\right)}} \lambda_{r}^{1 / 2}+\omega\left(r^{2} \log \frac{1}{r}\right),
$$

whenever $\left\|Q_{y}(u, r)\right\|_{L^{2}\left(\partial B_{1}\right)} \geq C$.

- Quadratic growth away from the free boundary

In [2], the norms of $\Pi_{y}\left(u, r / 2^{k}\right), k \geq 1$ are estimated in terms of the sum $\sum_{j=0}^{\infty} \lambda_{r / 2^{j}}$. If the norms of projections are unbounded, one obtain estimate (20) which implies convergence of the previous sum and hence boundedness of the projections. This is a contradiction. Similarly, in the general case the norms of $Q_{y}\left(u, r / 2^{k}\right), k \geq 1$ can be estimated by

$$
\sum_{j=0}^{\infty} \lambda_{r / 2^{j}}+\sum_{j=0}^{\infty} \omega\left(\left(\frac{r}{2^{k}}\right)^{2} \log \frac{2^{k}}{r^{2}}\right)
$$

Inequality (23) and Dini continuity imply

$$
\sum_{j=0}^{\infty} \omega\left(\left(\frac{r}{2^{k}}\right)^{2} \log \frac{2^{k}}{r^{2}}\right), \sum_{j=0}^{\infty} \lambda_{r / 2^{j}}<\infty
$$

if the norms of projections are unbounded. Furthermore, one completes the proof of the quadratic growth as in [2].

To verify that the above ingredients imply $C^{1,1}$ regularity, we split the analysis into two cases. If we are "far" from the free boundary, $u$ locally solves $\Delta u=g(x, u)$ so by Theorem $1.1 u$ is $C^{1,1}$. If we are close to the free boundary then $u$ solves $\Delta u=g(x, u)$ in a small ball $B_{d}(y)$ that touches the free boundary. We invoke (19) for $0<s<r=d / 2$ and the quadratic growth to obtain

$$
\begin{aligned}
\left\|Q_{y}(u, s)\right\|_{L^{2}\left(\partial B_{1}(0)\right)} & \leq\left\|Q_{y}(u, d / 2)\right\|_{L^{2}\left(\partial B_{1}\right)}+C\left(\left\|D^{2} v_{u(y)}\right\|_{\infty}+1\right) \\
& \leq C\left\|\frac{u(y+d / 2 x)}{d^{2} / 4}\right\|_{L^{2}\left(\partial B_{1}\right)}+C\left(\left\|D^{2} v_{u(y)}\right\|_{\infty}+1\right) \\
& \leq C+C\left(\left\|D^{2} v_{u(y)}\right\|_{\infty}+1\right) .
\end{aligned}
$$

for $s \leq d / 2$. 
So there exists a constant $C$ such that for all $y \in B_{1 / 2}$ there exist radii $r_{j}(y) \rightarrow 0$ such that

$$
Q_{y}\left(u, r_{j}(y)\right) \leq C
$$

We conclude via Lemma 2.6.

Acknowledgements We thank Henrik Shahgholian for introducing us to the regularity problem for semilinear equations. Special thanks go to John Andersson for valuable feedback on a preliminary version of the paper. E. Indrei acknowledges: (i) support from NSF Grants OISE-0967140 (PIRE), DMS-0405343, and DMS-0635983 administered by the Center for Nonlinear Analysis at Carnegie Mellon University and an AMS-Simons Travel Grant; (ii) the hospitality of the Max Planck Institute in Leipzig and University of Oxford where part of the research was carried out. L. Nurbekyan was partially supported by KAUST baseline and start-up funds and KAUST SRI, Uncertainty Quantification Center in Computational Science and Engineering.

Open Access This article is distributed under the terms of the Creative Commons Attribution 4.0 International License (http://creativecommons.org/licenses/by/4.0/), which permits unrestricted use, distribution, and reproduction in any medium, provided you give appropriate credit to the original author(s) and the source, provide a link to the Creative Commons license, and indicate if changes were made.

\section{References}

1. Agmon, S., Douglis, A., Nirenberg, L.: Estimates near the boundary for solutions of elliptic partial differential equations satisfying general boundary conditions. II. Commun. Pure Appl. Math. 17, 35-92 (1964)

2. Andersson, J., Lindgren, E., Shahgholian, H.: Optimal regularity for the no-sign obstacle problem. Commun. Pure Appl. Math. 66(2), 245-262 (2013)

3. Andersson, J., Weiss, G.S.: Cross-shaped and degenerate singularities in an unstable elliptic free boundary problem. J. Differ. Equ. 228(2), 633-640 (2006)

4. Badiale, M., Serra, E.: Semilinear elliptic equations for beginners, Universitext, Springer, London, Existence results via the variational approach (2011)

5. Cazenave, T.: An introduction to semilinear elliptic equations. Editora do IM-UFRJ, Rio de Janeiro, ISBN: 85-87674-13-7 (2006)

6. Figalli, A., Shahgholian, H.: A general class of free boundary problems for fully nonlinear elliptic equations. Arch. Ration. Mech. Anal. no. 1, 269-286 (2014)

7. Gilbarg, D., Trudinger, N.S.: Elliptic partial differential equations of second order. Springer, Berlin Heidelberg (2001)

8. Indrei, E., Minne, A.: Non-transversal intersection of free and fixed boundary for fully nonlinear elliptic operators in two dimensions. Anal. PDE. 9(2), 487-502 (2016). doi:10.2140/apde.2016.9.487

9. Indrei, E., Minne, A.: Regularity of solutions to fully nonlinear elliptic and parabolic free boundary problems. Annales de l'Institut Henri Poincare (C) Non Linear Analysis (2015). http://www. sciencedirect.com/science/article/pii/S029414491500044X

10. Koch, H., Nadirashvili, N.: Partial analyticity and nodal sets for nonlinear elliptic systems. arXiv: 1506.06224

11. Kovats, J.: Dini-Campanato spaces and applications to nonlinear elliptic equations. Electron. J. Differ. Equ. 1999(37), 1-20 (1999). http://ejde.math.txstate.edu/Volumes/1999/37/abstr.html

12. Lindgren, E., Shahgholian, H., Edquist, A.: On the two-phase membrane problem with coefficients below the Lipschitz threshold. Ann. Inst. H. Poincaré Anal. Non Linéaire 26(6), 2359-2372 (2009)

13. Petrosyan, A., Shahgholian, H., Uraltseva, N.: Regularity of free boundaries in obstacle-type problems, Graduate studies in mathematics, vol. 136. American Mathematical Society, Providence (2012)

14. Shahgholian, H.: $C^{1,1}$ regularity in semilinear elliptic problems. Commun. Pure Appl. Math. 56(2), 278-281 (2003)

15. Shahgholian, H.: Regularity issues for semilinear pdes (a narrative approach). Algebra i Analiz 27(3), 311-325 (2015)

16. Uraltseva, N.N.: Two-phase obstacle problem. J. Math. Sci. (New York) 106(3), 3073-3077 (2001). (Function theory and phase transitions) 